Proceedings of ASME Turbo Expo 2020

Turbomachinery Technical Conference and Exposition

GT2020

September 21-25, 2020, Virtual, Online

\title{
ZONAL DETACHED EDDY SIMULATION OF THE FAN-OGV STAGE OF A MODERN TURBOFAN ENGINE
}

\author{
Benjamin François, Raphaël Barrier \\ ONERA, The French Aerospace Lab \\ Aerodynamics, Aeroelasticity, Acoustics Department \\ CFD Turbomachinery Team \\ 8 rue des Vertugadins \\ 92190 Meudon, France
}

\author{
Cyril Polacsek \\ ONERA, The French Aerospace Lab \\ Aerodynamics, Aeroelasticity, Acoustics Department \\ Numerical Aeroacoustic Team \\ 29 Avenue de la Division Leclerc \\ 92320 Châtillon, France
}

\section{ABSTRACT}

The present article deals with the Zonal Detached Eddy Simulation of the fan module of a modern turbofan engine. The fan module, tested at the AneCom facility, is equipped with rotating fan blades and stationary outlet guide vanes $(O G V)$. The simulation was performed to capture the interaction of the turbulent fan wakes with the OGV walls. The final goal of this simulation is the prediction of the associated broadband noise, not adressed here. In this paper, only the aerodynamic aspects are treated. The simulation relies on a hybrid RANS/LES approach with a zonal strategy: the core airflow is treated in RANS while the bypass airflow is solved with the hybrid approach. Mesh criteria meeting both RANS/LES and acoustic requirements were fulfilled, leading to a mesh of 380 million cells. The simulation was performed during five revolutions and statistical convergence was reached. Inspections of the flow-fields highlight a consistent behaviour of the shielding function (border between RANS and LES solving areas) around the blade walls, at the trailing-edge and in the tip gap flow areas. Comparisons with performance and hot-wire measurements are also presented. Aerodynamic performance and radial evolution of averaged velocities on a plane in-between the fan and the OGV are well retrieved, both in shape and levels. For the turbulent quantities, the shape of the radial profiles are close to the measurements, with much better accuracy in the upper region compared to the RANS solution.

\footnotetext{
*Address all correspondence to this author : benjamin.francois@onera.fr
}

\section{NOMENCLATURE}

$\begin{array}{ll}\text { BC } & \text { Boundary Conditions } \\ \text { DDES } & \text { Delayed Detached Eddy Simulation } \\ \text { FBP } & \text { Fan Blade Passage } \\ \text { OGV } & \text { Outlet Guide Vanes } \\ \text { RANS } & \text { Reynolds Averaged Navier-Stokes } \\ \text { RMS } & \text { Root Mean Square } \\ \text { RSI } & \text { Rotor-Stator Interaction } \\ \text { URANS } & \text { Unsteady Reynolds Averaged Navier-Stokes } \\ \text { WMLES } & \text { Wall-Modeled Large Eddy Simulations } \\ \text { ZDES } & \text { Zonal Detached Eddy Simulation } \\ & \\ f_{d} & \text { Shielding function in the ZDES }\end{array}$

\section{INTRODUCTION}

Because of the significant reduction of tonal noise in modern turbofan, the broadband component of the overall perceived noise has become a growing contribution and is nowadays a major topic of concern for aircraft engine manufacturers. The present work adresses a hybrid RANS/LES methodology to predict this noise contribution on a fan module of a civil modern turbofan at approach conditions. Only the aerodynamic simulation and the associated results are presented here. In the fan module, a major contribution of the broadband noise is the interaction of the turbulent structures generated in the fan wakes 
with the outlet guide vanes (OGV) located downstream [1]. The turbulent structures are scattered by the OGV walls and radiate as acoustic waves in the upstream and downstream directions.

To estimate the in-duct radiated noise, the inherent mechanisms must be simulated or modelled. The whole aeroacoustic simulation process for predicting the broadband noise proceeds usually in three steps. First, the turbulent fan wakes need to be simulated either by RANS solving to get its main characteristics (velocity deficit, turbulent kinetic energy), either by turbulence scale resolved approaches to get unsteady, statistical and spectral quantities (RMS velocities, power spectral density). The choice of the approach is a trade-off between physics accuracy and numerical cost. The second step concerns the interaction of the turbulent wake with the vanes in order to get the unsteady pressure field at the vane wall. This interaction can be modeled by the means of analytical models in which the flow, the incoming wakes and the vanes geometry are strongly simplified or simulated with a turbulence scale resolved approach including both the fan and the OGV geometry. The latter strategy is adopted in the present paper and includes the two first steps aforementionned. The third step consists in computing the acoustic radiation from the pressure fluctuations within the engine duct using a Ffowcs-Williams Hawkings analogy. This part is not adressed here and will be presented in a dedicated paper [2].

Because of the high Reynolds number involved (around one million), turbulence scale resolved simulations of a fan blade or a fan stage of a turbofan engine require a significant computational effort. For this reason, such simulations remain scarce in the literature. Indeed, the high Reynolds number implies tremendous mesh refinement in the boundary layer for Wall-Resolved Large Eddy Simulations (WRLES). The use of wall law enables to reduce the numerical effort as proceed in Wall-Modeled LES (WMLES) but the mesh requirement still strongly increases with the Reynolds number. An alternative way is to resort to hybrid RANS/LES approaches in which the whole boundary layer is solved with a RANS modeling. Hence, their numerical cost is much less dependent on the Reynolds number. This simulation strategy is followed here and relies on the Zonal Detached Eddy Simulation from Deck et al. [3]. This approach has already been used by Bonneau et al. [4] on a turbofan for the prediction of broadband noise in which only a fan blade was simulated. The present work proposes to extend the work of Bonneau et al. by accounting for the OGV in the computational domain.

To the author knowledge, previous work based on turbulence scale resolved simulations for aeroacoustic purposes were performed by Leonard et al. [5] by means of WMLES on a NASA turbofan ${ }^{1}$. This work was continued by Arroyo et al. [6]. Hah and Romeo [7] performed similar WMLES simulation on the same configuration. The use of hybrid RANS/LES technique remains quite new for such applications.

${ }^{1}$ Source Diagnostic Test (SDT)
The objective of this paper is to evaluate the ability of the hybrid RANS/LES approach to predict a complex turbomachinery flow for aeroacoustic purposes. First, the paper presents the main features of the fan geometry and operating point. Then, several aspects of the CFD strategy such as the methodology, the mesh critera, the boundary conditions and the numerical parameters are discussed. Third, analysis of the convergence of the simulation are addressed. Fourth, three-dimensional analysis of the flow inspecting the consistency of the ZDES solution are carried out. Finally, comparisons with hot-wire measurements performed in AneCom test falicity [8] are discussed.

\section{Geometry and Operating Point}

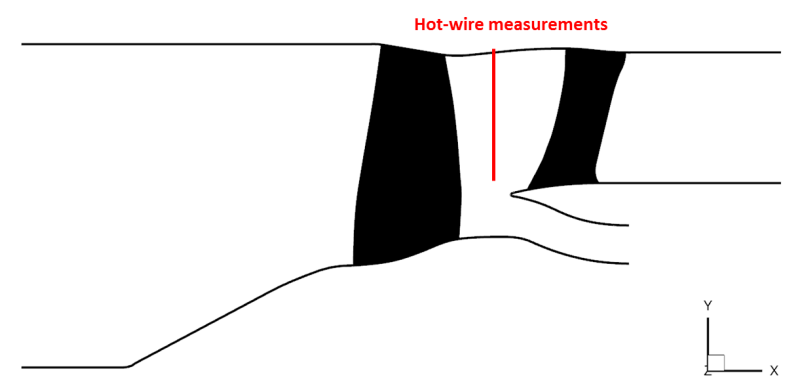

FIGURE 1. Meridional view of the fan module with the hot-wire probes.

Figure 1 presents a meridional view of the fan-OGV stage. Its main original features is the short interstage distance. The configuration is equipped with 20 fan blades and 44 outlet guided vanes (OGV). The configuration is studied at rig-scale (the outer radius of the fan is $0.42 \mathrm{~m}$ ). Because the bypass ratio is around six, the rotor-stator interaction (RSI) with the Inlet Guide Vanes (IGV) can be assumed negligible compared to the bypass RSI contribution. As a result, the IGV are not accounted for in the simulation. The configuration is studied at the approach conditions (50\% Nn, namely $3828.4 \mathrm{rpm}$ ) for which the RSI noise is dominant.

\section{Methodology}

\subsection{Zonal Detached Eddy Simulation}

The Zonal Detached Eddy Simulation (ZDES) developped by Deck et al. [3] and implemented in the elsA code [9] is used here. This formulation offers the possibility to treat different parts of the computational domain with a specific DES formulation or a full URANS solving. This approach has already been used on aircraft, space launchers, jet flows and turbomachinery $[4,10,11]$. The zonal aspect enables to alleviate the computa- 
tional cost compared to other DES techniques with areas treated with a RANS solving, requiring a reduced mesh density. Also, such approach offers a large flexibility for the user to choose the most suitable DES formulation with respect to the flow physics to deal with. The present paper only describes the DES mode 2 (refering to Deck et al. [3]) used in the simulation and the reader can refer to the aforementionned paper for further informations.

The DES mode 2 allows the boundary layer to be solved with an URANS treatment, the remaining part being treated by a LES solving. To detect whether the computed cell is in the attached boundary layer or not, a shielding function $f_{d}$ is evaluated and reads:

$$
f_{d}=1-\tanh \left[\left(8 r_{d}\right)^{3}\right], \quad r_{d}=\frac{v+\tilde{v}}{\sqrt{U_{i, j} U_{j, i}} \kappa^{2} d_{w}^{2}}
$$

where $\tilde{v}$ is the eddy viscosity solved in the equation of the Spalart model $[12]^{2}, v$ the molecular viscosity, $U_{i, j}$ the velocity gradient, $\kappa$ the Karman constant, $d_{w}$ is the distance to the wall. The parameter $r_{d}$ is equal to one in the logarithmic area of the boundary layer and vanishes at the edge of the boundary layer. Consequently, the function $f_{d}$ equals zero in the boundary layer and actives the URANS mode. Outside the boundary layer, it equals one and allows the switch to the LES mode. The DES mode 2 from Deck et al. presents similarities with the Delayed Detached Eddy Simulations (DDES) from Spalart et al. [13]. The main difference concerns the choice of the mesh length and on the user defined function $f_{d 0}$ between values ranging from 0.75 and 0.99 . Deck et al. made comparisons between the DDES and the DES mode 2 on various applications (mixing layer, backward facing step, airfoil). They highlight that ZDES keeps the strong assets of DDES such as the treatment of the attached boundary layer while improving other aspects such as reducing the delay in the formation of the instabilities.

\subsection{Repartition of RANS and DES zones}

The objective is to simulate the interaction between the fan turbulent wake with the OGV in the bypass airflow. Consequently, the core airflow can be solved with an URANS solving, reducing the mesh requirements in this area. Also, the upstream turbulence rate measured in the experimental facility upstream the fan is very low (around $0.3 \%$ ) [8]. Hence, the noise associated to the impingement of the infinite inflow turbulence on the fan is negligible compared to the RSI noise. Thus, no turbulence solving is necessary upstream the fan and this area can be treated with URANS solving. All the remaining areas of the computational domains are treated with the DES formulation described in section 2.1. An illustration of the RANS/DES zones on single channel computational domain is given in Fig. 2.

\footnotetext{
${ }^{2}$ In the classic DDES from Spalart [13], the variable $v_{t}$ is used.
}

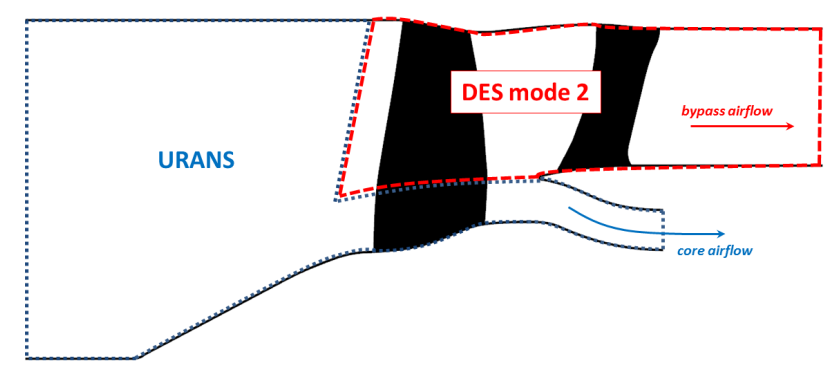

FIGURE 2. Repartition of the RANS and DES areas in the computational domain.

\subsection{Modification of the geometry}

2.3.1 Motivation To make ZDES accessible in terms of numerical cost, only a few interblade channels are simulated. The current number of blades ( 20 for the fan, 44 for the OGV) is unfavorable to reduce the computational domain to a few interblades channels. For unsteady RANS simulations, classic phaselag boundary conditions allows the reduction to one channel. Because they are based on the usual Fourier decomposition, they are unadapted for the convection of broadband structures . Consequently, a more favorable option is to change the blade number of the OGV to get a suitable divisor and allow the use of periodic boundary condition instead. Even if the periodicity is still questionnable for the turbulence structures between adjacent channels, the spectral content is believed to be conserved through these periodic boundary conditions. This strategy has already been justified and used by several authors on the NASA Source Diagnostic Test configuration for broadband noise predictions [5-7].

2.3.2 OGV Rescaling For the present application, the number of the OGV is thus reduced from 44 to 40 . To keep the same flow pattern, the solidity is kept constant meaning that the chord is increased by $10 \%$, the leading-edge position is unchanged. Such a blade number modification allows to reduce the computational domain to one fan channel and two OGV channels.

Figure 3 illustrates the new geometry for the rescaled OGV. The unchanged geometrical parameters are the solidity, the leading-edge position, its sweep and lean angles, the thicknessto-chord and angles law along the streamwise variable and inner and outer duct walls. The modified parameters with respect to the original geometry are the number of blades (44 to 40), the chord $(+10 \%)$, the aspect ratio and the thickness.

2.3.3 Aerodynamic impact of the OGV Rescaling RANS simulations of the fan-OGV configuration with the nom- 


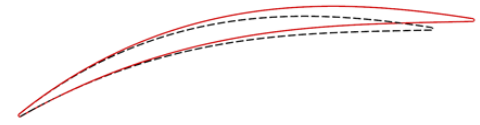

FIGURE 3. Modification of the OGV geometry.

inal number of OGV and with the modified number of rescaled OGV were performed at the same massflow. Inspection of the absolute flow angles, absolute Mach number at position upstream and downstream the OGV show identical radial evolutions (for conciseness, only the flow angles downstream are shown in Fig. 4). Same conclusions were drawn regarding the pressure maps distribution on the walls. The conservation of the solidity is a key aspect in the OGV modification. First, it enables to get a similar mean velocity field to convect the fan wake through the vanes. Second, a modification of solidity can affect the unsteady pressure fluctuation due to wake interaction, as shown by Posson et al. [14].

The present comparison actually ensures that the mean flow around the OGV remains similar with the modification of the geometry.

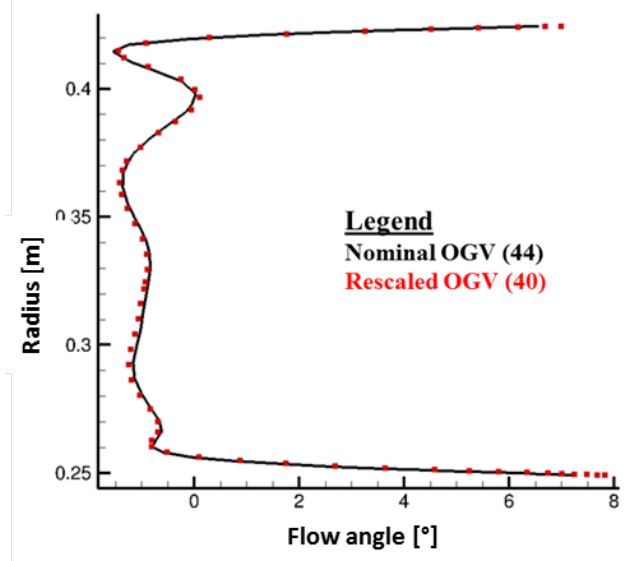

FIGURE 4. Comparison of the flow angles at half-chord downstream the OGV

\section{Numerical setup and Boundary Conditions 3.1 Computational domain}

The computational domain is composed of one fan and two OGV inter-blade channels. The fan and the OGV domains are solved in their respective frame of reference. The inlet of the computational domain is located at four fan chords upstream of the fan leading-edge while the outlet is located at three vane chords downstream of the vane trailing-edge.

\subsection{Boundary Conditions}

The modification of the OGV geometry mentionned in the previous section enables to get the same azimuthal periodicity with one fan blade and two vanes. As a consequence, periodic boundary conditions can be used for the azimuthal border of the computational domain for the unsteady simulation. At the interface between the rotating domain of the fan and the fixed domain of the vanes, a sliding mesh treatment is performed. It relies on interpolation of the state vector of a donor grid on a receive grid and then on a half-sum between the donor and the receiver cells from both domain on the conservative variables.

At the inlet of the computational domain, stagnation pressure and enthalpy are imposed with the values measured during the experimental campaign. The absolute velocity direction is imposed and is axial. For the outlet of the core and the bypass airflow, a radial equilibrium is imposed. The inlet and outlet BC are combined with sponge layer to limit spurious reflections (see further details in Sec. 4.2.2).

\subsection{Numerical parameters}

The time scheme is ruled by an implicit second order scheme based on a Newton algorithm. The timestep has been set to $3.2 \times$ $10^{-7} s$. The choice was mainly driven by acoustic $\mathrm{CFL}^{3}$ number considerations and computational cost (the selected timestep is small enough to capture the turbulent structures at stake). It corresponds to a convective CFL around 36 number in the smallest cells and of 0.35 for cells the length of which is $0.5 \mathrm{~mm}$. This timestep samples the fan blade passing periods by 2400 and the revolution by 48000 . 5 sub-iterations are used in the inner-loop of the time scheme and the residuals decrease by one order of magnitude.

The convective flux discretization is treated with the AUSM (Advection Upstream Splitting Method) scheme with a third order limiter. The turbulence model used is the Spalart model [12].

The simulation is performed on the supercomputers from CINES $^{4}$. The simulation is done on 600 cores. Five full revolutions were simulated.

\section{Mesh}

\subsection{Mesh criterion}

Two sets of criteria were used to build the present mesh. The first relies on internal best practices concerning the use of the DES mode 2. The cell size must verify $\Delta x^{+}, \Delta y^{+}, \Delta z^{+}<200$ in the free shear flow areas. It implies mesh size lower than 0.6 $\mathrm{mm}$. The first wall mesh size verifies $\Delta y^{+} \leq 1$.

The second criterion relies on the aeroacoustic mechanism at stake. The impinging turbulent wake from the fan blade can be modelled by a set of hydrodynamic waves. The impingement

\footnotetext{
${ }^{3}$ Courant Friedrichs Lewy

${ }^{4}$ French National Computing Center
} 


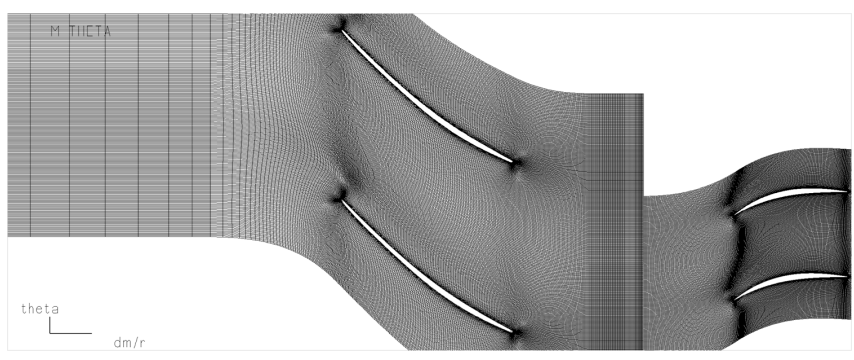

(a) Fan inter-blade channel

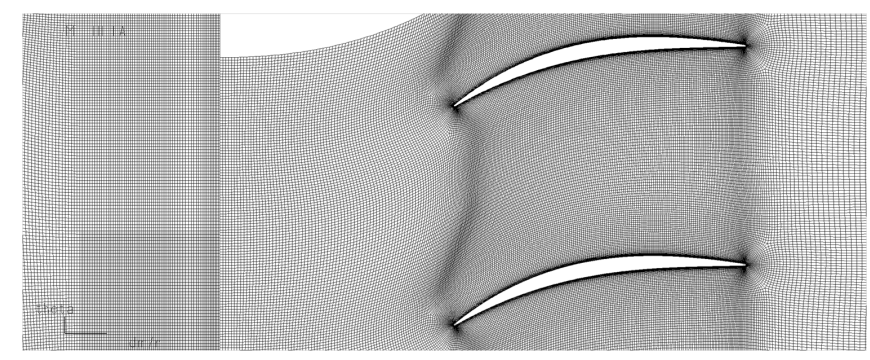

(b) Vane inter-blade channel

FIGURE 5. Blade-to-blade view at mid-height of the OGV span. Meshes with one point over two are shown in the pictures.

of each hydrodynamic wave, also called gust, on the vane will generate acoustic waves with the same frequency. Among the different waves at stake and for the Mach number of the considered flow, the gust wavelength is the most stringent. Its expression reads:

$$
\lambda_{h}=\frac{U_{c}}{f}
$$

where $U_{c}$ is the local convection speed and $f$ is the frequency. Preliminary RANS simulations show that in the interstage area, the absolute velocity ranges from $100 \mathrm{~m} / \mathrm{s}$ and $140 \mathrm{~m} / \mathrm{s}$. Accounting for the smallest velocity value and sampling the wavelength by 20 points leads to a mesh size of $0.25 \mathrm{~mm}$ at $20 \mathrm{kHz}$. Such cell size would imply for the intended computational domain a mesh of 3 billions cells. Finally, the selected cell size was $0.5 \mathrm{~mm}$ and ensure a sampling of 20 points at $10 \mathrm{kHz}$.

\subsection{Mesh characteristics}

4.2.1 Repartition of points The mesh was build to meet the criteria mentionned above. The mesh used is structured and multi-blocks. The total cell number is equal to 380 millions: 180 for the fan domain, 100 for each OGV channel. The number of cells in the radial direction is 85 in the core airflow, 41 around the radial position of the splitter and 645 in the bypass airflow. 45 cells are used in the tip gap. In the inter-blade channel, 280 cells are used in the streamwise direction from the leading-edge to the trailing-edge while 380 cells are used in the azimuthal direction. Downstream of the fan and up to the fan/OGV domain interface, the number of points in the azimuthal direction is around 300. In the OGV domain, the number of cells in the radial direction is 625 . In the inter-blade channel, 400 cells are used in the streamwise direction from the leading-edge to the trailing-edge while 264 cells are in the azimuthal direction. Upstream of the OGV, the number of cells in the azimuthal direction reduces to 160 points. The first mesh size is three micrometers and correspond to $y^{+}$values equal to 1 at the cell center. Sectional views are given in Fig. 5.
4.2.2 Buffer areas Near the inlet and the outlet, block meshes with increasing cell size in the axial direction are used to avoid numerical reflections on the boundary conditions. Their expansion ratio remains lower than 1.2 for these cells to prevent additional spurious acoustic reflections. The largest cell size upstream of the fan is three centimeters while it is equal to two centimeters downstream of the OGV.

\section{Initialization setup}

To reduce the numerical transient in the ZDES, a preliminary set of RANS and then URANS simulations were performed. The URANS simulation was run until the massflow rate was converged, namely two complete revolutions.

\section{Convergence of the simulation}

\subsection{Numerical transient}

The determination of the numerical transient is here detected by the convergence of the time-averaged quantities (over a large time-width with respect to the blade period) of the flow quantities either global (massflow) or local (axial momentum on a numerical probes). Figure 6 gives an illustration by showing the evolution of the bypass air mass flow non-dimensioned by its final value at $t=96 \mathrm{FBP}^{5}$. Both the instantaneous signal and the timeaveraged one over ten FBP are shown. The abscissa axis is given in fan blade passage ( 20 equals a complete revolution). From the URANS simulation, the massflow shifts slighly because of the flow solution is very different between the RANS and the ZDES in the flow separated areas. The massflow curve shows that the massflow is stabilized after one and half revolutions (variations of the time-averaged quantity on ten fan blade passages are lower than $0.1 \%$ ). Flow quantities such as the axial momentum and the static pressure on numerical probes (not shown here for conciseness) meet the same requirement. The conjoint analysis of different time-averaged flow quantities indicates the numerical transient last one and half revolutions.

\footnotetext{
${ }^{5}$ This nondimensionalization gives direct access to the time-variation of the signal in percent.
} 


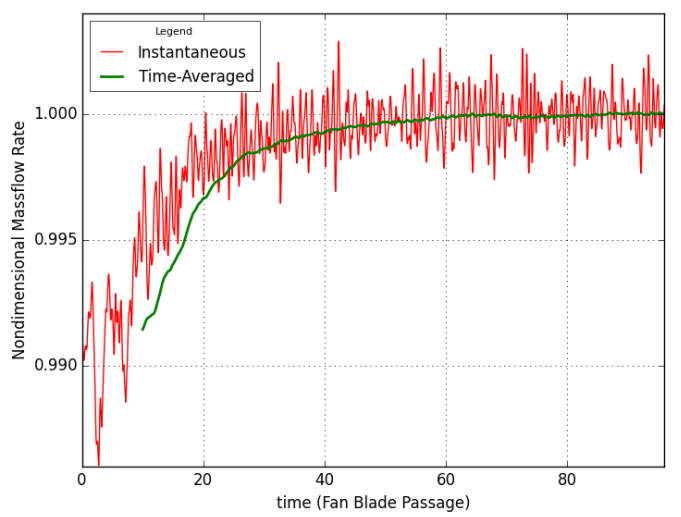

FIGURE 6. Convergence of the bypass air massflow rate at the outlet of the computational domain, nondimensioned by the massflow rate at $t=96 \mathrm{FBP}$.

\subsection{Statistical convergence}

6.2.1 Cyclostationnary signal For the convergence of the statistics, the numerical transient of the signal is removed from the complete signal. Only the portion from $t=30 \mathrm{FBP}$ to $t=96 \mathrm{FBP}$ is used to perform the statistics. Because the flow quantities exhibits strong deterministic signatures due to fanOGV interaction in both the fan and the OGV computational domain, a cyclostationnary decomposition of the signal is used. Inspired by the pioneer idea of Hussain and Reynolds [15], the signal is split into a deterministic and a stochastic part:

$$
u(x, t)=u_{\text {cyclo }}(x, t)+u_{s t o c}(x, t)
$$

where $u_{\text {cyclo }}(x, t)$ contains the deterministic variation of the signal and its time averaging and $u_{\text {stoc }}(x, t)$ the remaining part of the turbulent signal. The cyclostationnary signal $u_{\text {cyclo }}(x, t)$ is performed with the following number of fan blade passages:

$$
N(t)=E\left(\frac{t-t_{0}}{T_{B P}}\right)
$$

where $t_{0}$ is the duration in time of the numerical transient, $T_{B P}$ is the period of one blade passage. This relation enables to see the progressive convergence of the cyclostationnary signal. The last period of the cyclostationnary signal is then used to compute the fluctuating part. In the fan domain, the OGV blade passage period is used while in the OGV domain, the fan blade passage period is used. A direct application is performed on the axial momentum signal on the numerical probe located at mid-height near the OGV leading-edge. Figure 7 presents the instantaneous signal during six FBP and the cyclostationnary signal. In the

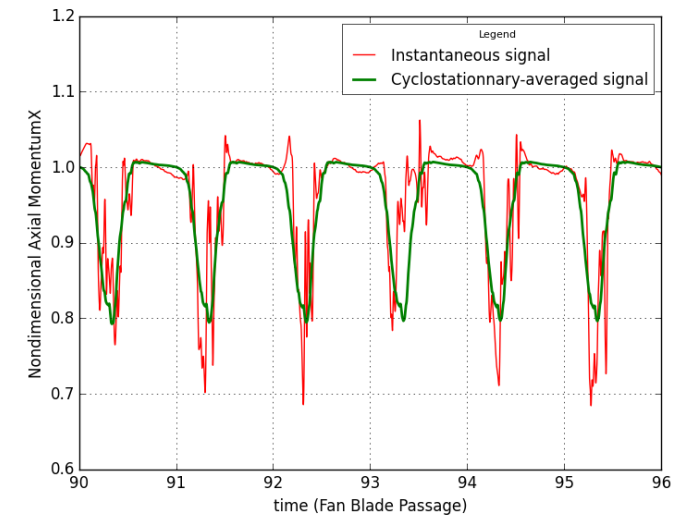

FIGURE 7. Instantaneous and cyclostationnary signals of the axial momentum on a numerical probe at mid-height, near the OGV leadingedge

cyclostationnary signal, the stochastic part of the signal is filtered. The identical shape of this signal between the successive fan blade periods indicate that the signal is phase-averaged. Accurate repeatability of the phase-averaged FBP signatures is a good indicator of the convergence of the periodic contributions to be assessed in view of cyclosationnary analyses. In contrast, the instantaneous signal exhibits different velocity deficits for each fan blade period due to the stochastic nature of turbulence.

The phase-averaged property of the signal indicates that the time signal (without the numerical transient) is long enough to get converged cyclostationnary averages and thus reliable informations concerning the determinist part of the fan-OGV interaction.

6.2.2 Stochastic part of the signal The stochastic part of the signal is obtained by substracting the cyclostationnary average to the instantaneous signal $u(t)$. To evaluate its convergence over the simulation, the Root Mean Square treatment over moving windows is introduced:

$$
\begin{aligned}
\overline{u_{s t o c}}(t) & =\frac{1}{M(t)} \sum_{n=1}^{N(t)} u_{s t o c}(t) \\
u_{s t o c-R M S}^{2}(t) & =\frac{1}{M(t)} \sum_{n=1}^{M(t)}\left(u_{s t o c}(t)-\overline{u_{s t o c}}(t)\right)^{2} \\
M(t) & =E\left(\frac{t-t_{0}}{\Delta t}\right)
\end{aligned}
$$

where $\Delta t$ is the timestep, $t_{0}$ is the duration of the numerical transient. The window size $M(t)$ increases with the time variable. Figure 8 presents the evolution of the variable $u_{s t o c-R M S}^{2}$ with 


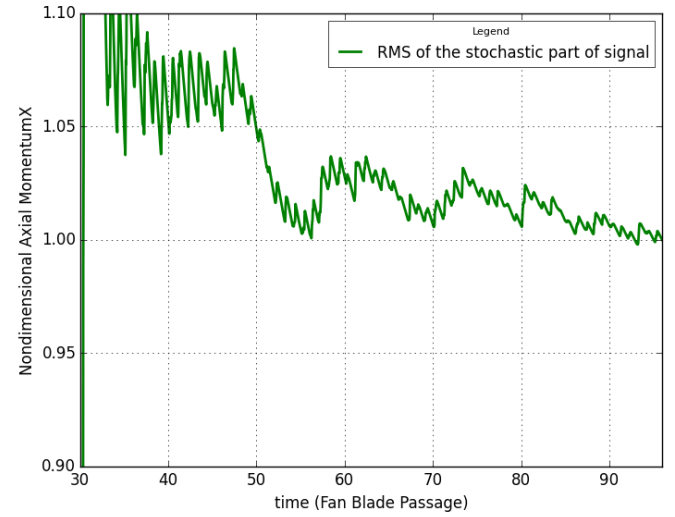

FIGURE 8. Convergence of the Root Mean Square (RMS) of the stochastic part of the axial momentum signal on a numerical probe at mid-height close to the OGV leading-edge

respect to the time variable to evaluate the convergence of the stochastic part. The same nondimensionalization as Fig. 6 is used. As shown in Fig. 8, during the last revolution (20 fan blade periods), the RMS variation of the stochastic part of the signal are less than a few percents, which is satisfactory.

\section{Aerodynamic analysis}

This section focuses on the analysis of the aerodynamics flow-field and on the consistency of the flow solution obtained with the ZDES approach.

\subsection{Zonal solving}

Figure 9 presents a visualisation of the instantaneous flowfield around the fan blade : surfaces of iso-values of the Q criterion colored by the axial momentum are displayed. It illustrates the zonal solving with the ZDES. The core airflow is treated in RANS and only very large turbulence structure are present such as an horseshoe vortex at the intersection of the fan blade and the hub. The bypass airflow is treated with the DES mode 2 (see Section 2.1). Because the flow separates at the leading-edge of the fan for this operating point, small turbulent structures are generated there and are convected downstream. The high grid density enables to capture small turbulent structures.

\subsection{Convection of the fan wakes through the OGV}

The main source of broadband noise is the interaction of the turbulent structures from the fan wake with the OGV walls. An important key in this simulation is to ensure that the turbulence is properly convected from the fan through the OGV. Figure 10 presents fields of vorticity magnitude on a developed blade-toblade surface. The vorticity fields are consistent: the fan wakes

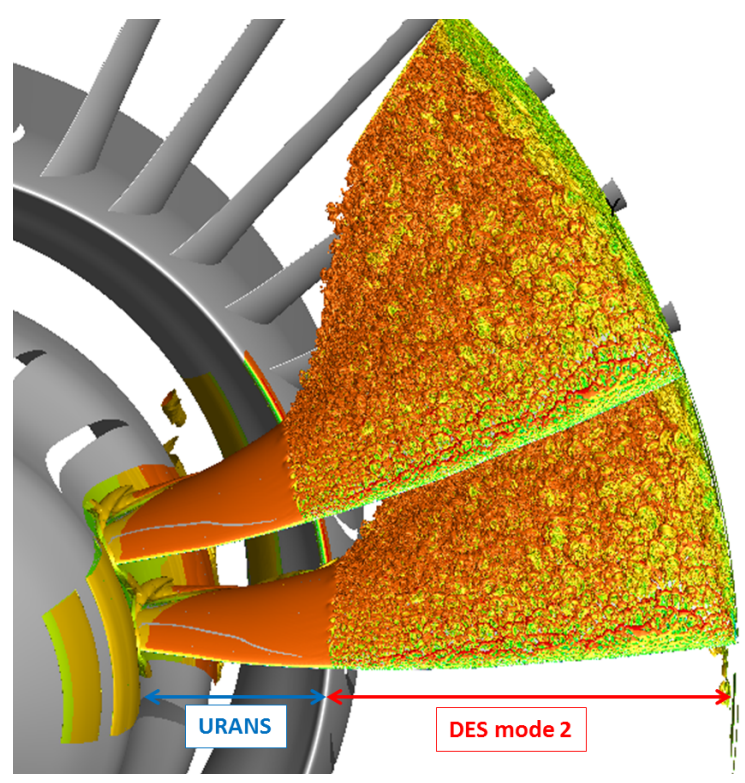

FIGURE 9. Illustration of the zonal solving with ZDES by the visualisation of iso-value surfaces of the $\mathrm{Q}$ criterion around the fan blades.

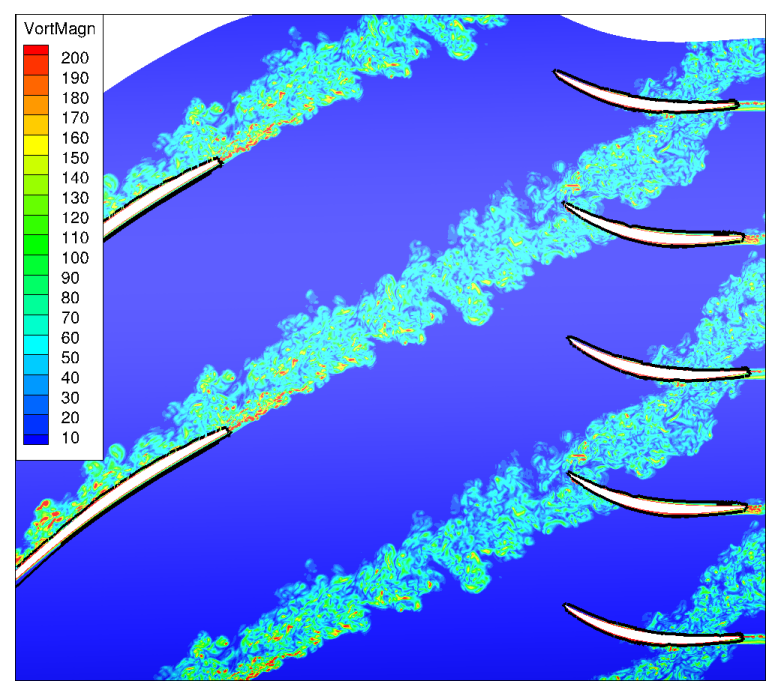

FIGURE 10. Visualisation of vorticity magnitude fields on a developed blade-to-blade surface at $50 \%$ of the OGV span. The black lines denote $f_{d}=0.95$.

are convected without noticeable attenuation through the stage interface and the periodic boundary conditions.

Figure 10 depicts in thick black lines the areas in which the shielding function $f_{d}$ equals 0.95 , defining thus the border between the RANS solving (in the boundary layer) and the LES solving (in the free shear flow areas). For both fan and OGV blades, this RANS solving is consistent and seems qualitatively to encompass the whole boundary layer. A noticeable point is the 
rapid switch from RANS to LES at the trailing-edge of the blade (visible here only on the fan blades) ${ }^{6}$. The RANS boundary layer velocity profile degenerates into turbulent eddies due to a good detection of a free-shear flow area and the specific features of the ZDES described in the work of Deck et al. [3]. Similar conclusions with the same approach were drawn by Gand on a flow separation on a spoiler [16], Verrière et al. on a jet flow [17] and by Mockett et al. on free shear flows [18].

A second noticeable point concerns the shielding function shape around the OGV walls: the interaction of the turbulent structures from the fan wake with the OGV do not (qualitatively) degrade the shielding of the boundary layer. This demonstrates a good robustness and maturity of the approach for turbomachinery flows.

\subsection{Tip gap flow}

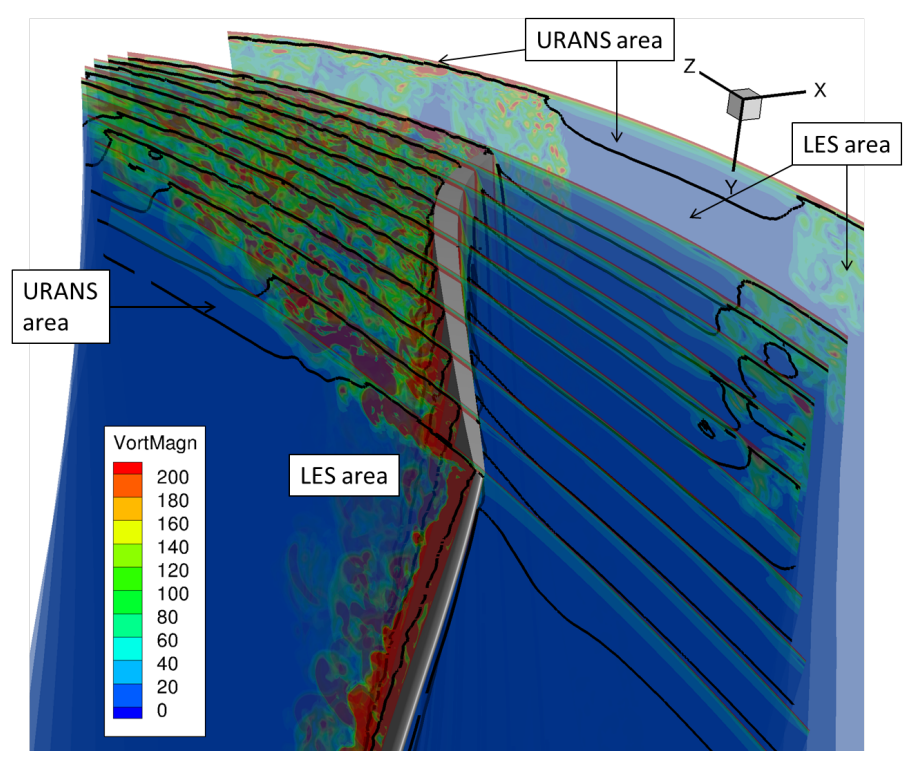

FIGURE 11. Visualisation of vorticity magnitude fields on multiple surfaces of constant X coordinates. The black lines denote $f_{d}=0.95$.

Tip gap vortices interact with vanes and contributes to the generation of broadband noise. Figure 11 shows that upstream of the fan, the near wall (fan outer shroud) area treated in RANS mode is $7 \mathrm{~mm}$ thick. Consequently, the tip gap, the size of which is $0.78 \mathrm{~mm}$, is fully immerged into a RANS area.

It could be expected that with hybrid RANS/LES approaches, only RANS-shape vortices would be generated. However, inspection of the vorticity magnitude and of the shielding function (lines at $f_{d}=0.95$ ) on Fig. 11 gives an other perspective.

\footnotetext{
${ }^{6} \mathrm{~A}$ delayed switch to LES is a current issue with DES-like approaches.
}

On the suction side, vortices arise due to the pressure difference between the pressure side and the suction side of the blade. The sensor $f_{d}$ detects the generation of these vortices and switches to the value of one. The convection of the tip gap vortices towards the RANS-solved area makes the model turn into LES. Along the chord, tip vortices keep developing in the tangential direction. Near the casing, we observe two distinct areas: a thick RANS area capturing the boundary layer initiated at the inlet of the computational domain and not perturbated by the tip vortices and a very thin RANS area. Inspection of several axial planes in Fig. 11 show that the thick RANS area reduces in the downstream axial planes. It fully disappears in the axial places further downstream (not shown here for conciseness).

\subsection{Conclusions}

The overall analysis of the aerodynamics flow underlines a consistent behavior of the RANS/LES approach applied to a complex turbomachinery flow. The key aerodynamic phenomenon responsible for the generation of broadband noise such as the wake interaction in the secondary airflow are qualitatively well captured.

\section{Comparison to measurements}

Measurements were performed on the selected fan configuration at the studied operating point. Hot-wire measurements in between the fan and the OGV for the three directions of velocity are available. The reader can refer to the paper of Meyer et al. [8] for further informations.

\subsection{Aerodynamic performances}

\begin{tabular}{lcc}
\hline & Measurements & ZDES \\
\hline Core massflow $[\mathrm{kg} / \mathrm{s}]$ & 6.41 & 6.44 \\
\hline Bypass massflow $[\mathrm{kg} / \mathrm{s}]$ & 48.76 & 49.26 \\
\hline Bypass pressure ratio & 1.108 & 1.106 \\
\hline
\end{tabular}

TABLE 1. Comparison of aerodynamic performances

Table 1 highlights that the aerodynamic performances are quite well retrieved by the simulation. Discrepancies on the bypass massflow are due to the difficulty to monitor the massflow with the DES solving : the numerical transient is very long (see Fig. 6) and the counter-pressure matching the experimental massflow and estimated from the URANS solution needed to be recalibrated. 


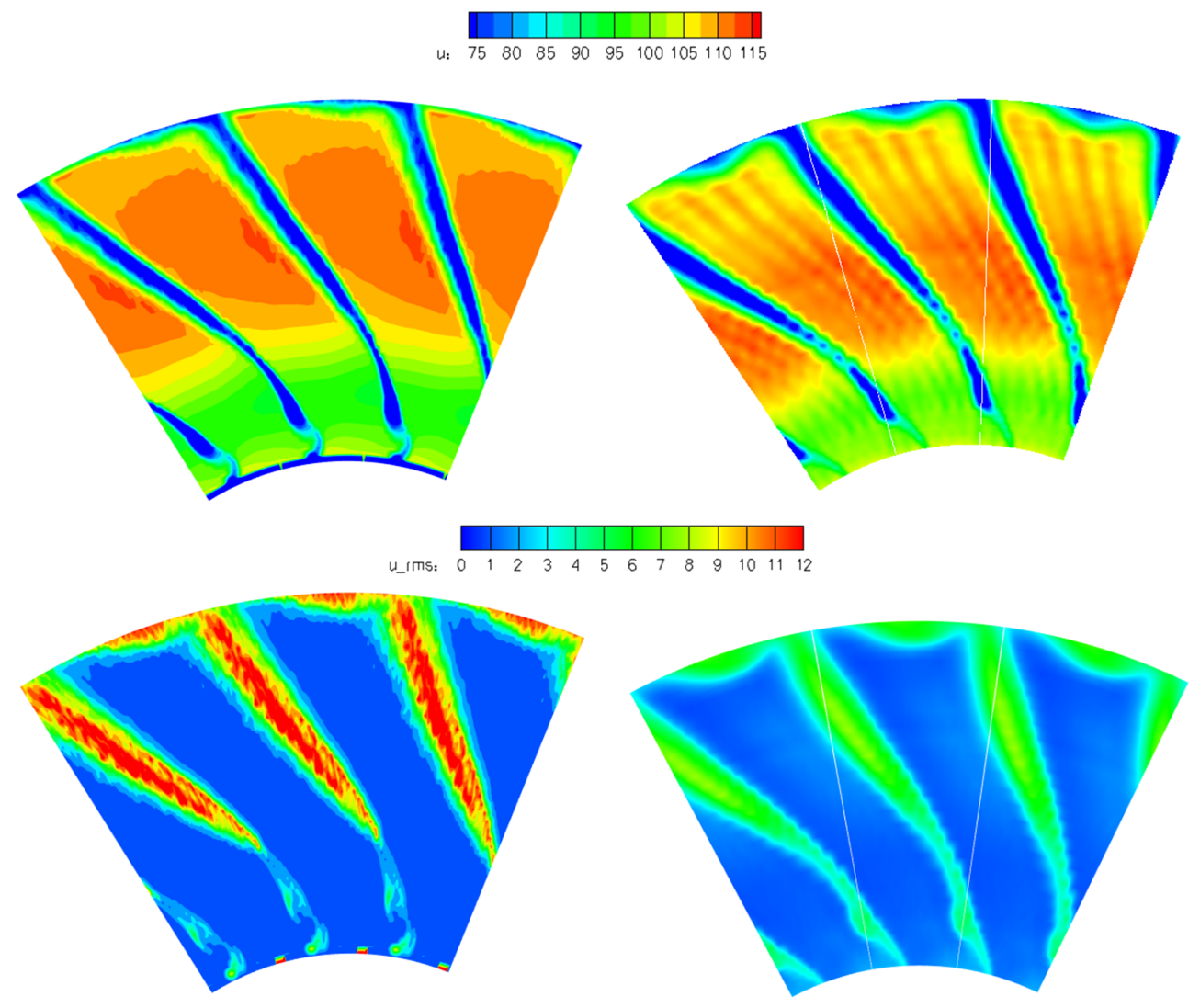

FIGURE 12. Maps of axial velocity flow fields at HW1 plane with ZDES (left) and measurements (right) - Three blade channels are displayed: Cyclostationnary quantities (top) and RMS quantities (bottom)

\subsection{Two-dimensional map visualisations and wake features}

The ZDES solution has been extracted over a cross-section at HW1 position in order to perform mean flow and wake analyses, by splitting periodic and fully turbulent contributions, and to make comparisons with available measurements. Present results are focused on phase-locked averages (cyclo-stationnary fields) and statistical quadratic averages (RMS turbulent fields). The turbulent structures are essentially present in the wakes and can be easily removed using a phase averaging triggered to the FBP leading to the ZDES plot of Fig. 12 (top left), restricted to three blade channels for a better appraisal of differences with the experiment (top right). RMS fields of turbulent velocity (axial component) are compared in Fig. 12, bottom. The shape and width of the wakes are well reproduced by the simulation. Major discrepancies are observed on the levels of the turbulent velocity (more intense in the ZDES solution). In fact, this level offset was recently attributed to the limited bandwidth of the hot-wire probes (cut-off frequency around 7-8 $\mathrm{kHz}$ ). This leads to an underestimation of the RMS values in the experiments. One should note that the steep level attenuation in the foot region of ZDES solution (Fig. 12, bottom left) is due to the transition to RANS solved region (as sketched in Fig. 2).

\subsection{Mean flow and turbulence radial profiles}

The above 2D fields have been circumferentially averaged in order to perform more accurate radial profile comparisons. Axial and tangential components of mean velocity provided by ZDES and experiment are plotted in Fig. 13 left and right, respectively. The shapes of the radial profiles are well predicted. The average discrepancies are around $2 \%$ (with maximum at $4 \%$ for some radius).

Similar comparisons of radial profiles have been realized for the three components (axial, tangential, radial of turbulent velocity leading to RMS values plotted in Fig. 14. Only the radial section concerning the secondary airflow (DES mode 2 solving) is shown. Firstly, due to the bandwidth issue with the hot-wires (HW), a calibration of the measured data is proposed in Fig. 14 by applying a 1.5 factor correction (estimated from Parseval identity and integration of velocity PSD up to $8.5 \mathrm{kHz}$, cut-off frequency of the HW probes). A satisfactory agreement is achieved with the calibration. Secondly, discarding the hub 

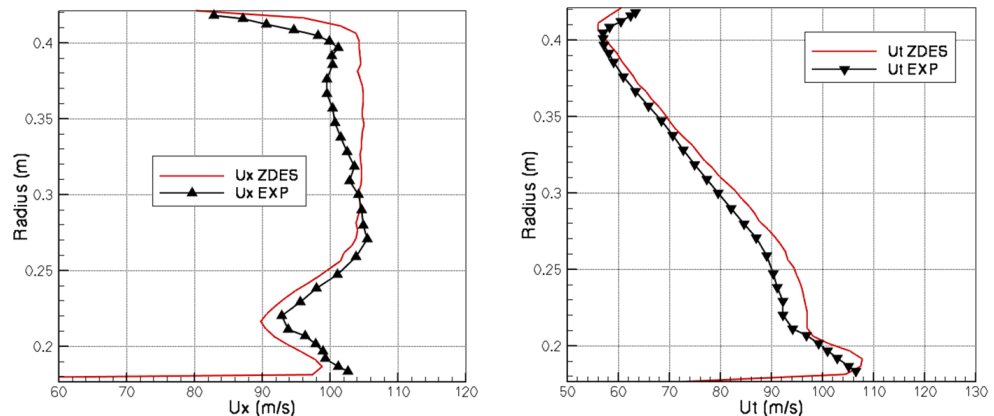

FIGURE 13. ZDES and experimental radial profiles: axial (left) and tangential (right) mean velocity
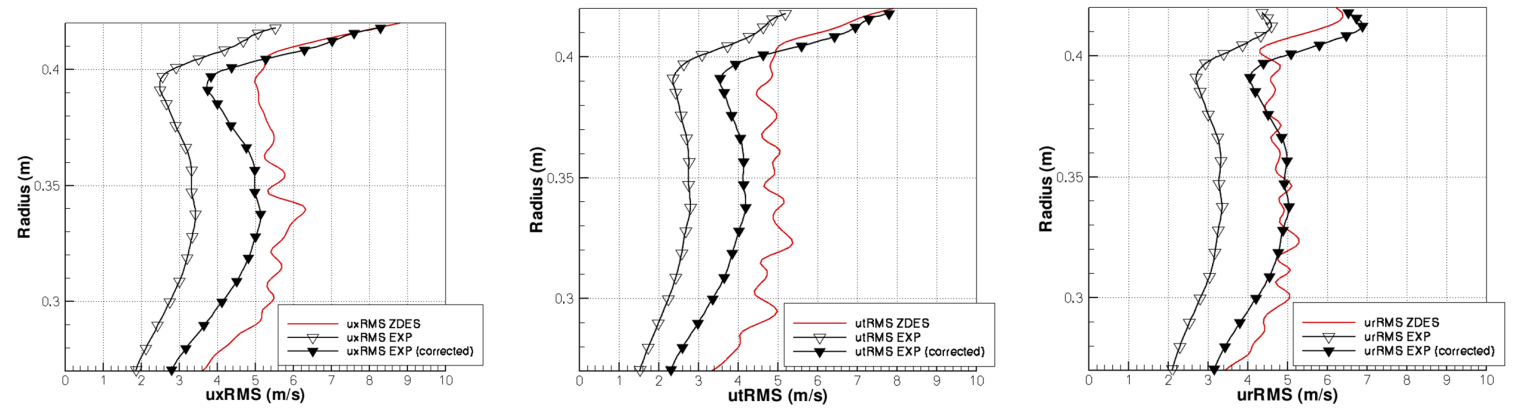

FIGURE 14. ZDES and experimental radial profiles: axial (left), tangential (center) and radial (right) RMS turbulent velocity.

region (roughly up to $0.28 \mathrm{~m}$ radius) that is not able to capture turbulent eddies due to the RANS modelling in this area, the radial evolutions are in a good agreement up to the tip, even if the ZDES levels are still a bit higher compared to the corrected experiment. Thirdly, these comparisons highlight that the turbulence is nearly isotropic (in the sense of spatial energy) as quite similar profiles and levels are observed for the three components, both from ZDES and measurements.

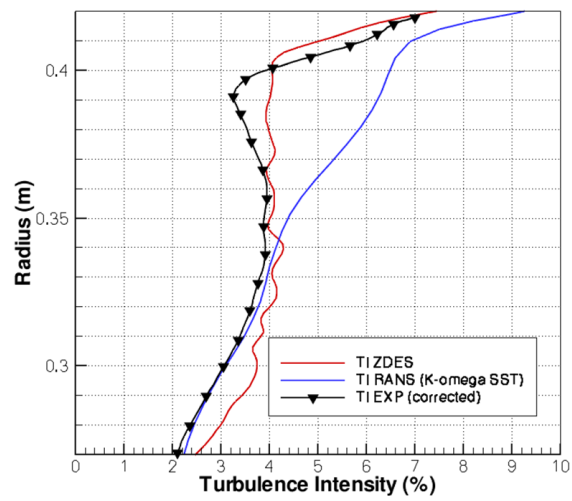

FIGURE 15. Radial profiles of turbulence intensity from RANS, ZDES and experiment. Only the radial section with the secondary airflow is shown.
A last analysis is proposed in Fig. 15 that compares turbulence intensity profiles issued from CFD and experiment. An additional full RANS solution obtained from a complementary calculation using a $k-\omega$ Menter SST model is shown. The ZDES solution and measurements provide a satisfactory agreement whereas the RANS solution present large discrepancies on the levels and the shape above $50 \%$ span $(r=0.35 \mathrm{~m})$.

\section{Conclusions}

A Zonal Detached Eddy Simulation approach has been applied to a turbofan stage to simulate the interaction of the turbulent fan wakes with the OGV. The final goal of this simulation is the prediction of the associated broadband noise, not adressed here. Only the aerodynamic aspects were treated in the present paper. To limit the numerical efforts, the number of the OGV blades has been modified to allow a significant reduction of computed inter-blade channels while preserving the main aerodynamic performances.

The ZDES was performed on five revolutions and statistical convergence (thanks to analysis of numerical controller probes) was achieved. Inspection of the flow field highlights a very consistent behaviour of the shielding function (border between RANS and LES solving areas) and a correct convection of the broadband turbulent structures to the OGV.

A comparison with performance and hot-wire measurements 
was presented. Aerodynamic performance and radial evolution of averaged velocities on a plan in-between the fan and the OGV are well retrieved, both in shape and levels. For the turbulent quantities (RMS, turbulence intensity), the shape of the radial profiles are close to the measurements. The predicted shape with the ZDES is better than the RANS one.

Following work is focusing on acoustic post-processing [2], covering the coupling with Amiet-based code using turbulent wake characteristics and direct sound radiation from OGV sources using a FWH-based code, as well as acoustic comparisons with measurements from available microphones. Comparisons of these simulations results with those from the EU partners performing RANS and scale-resolved turbulence simulations are also planned.

\section{ACKNOWLEDGMENT}

The presented work was conducted in the frame of the project TurboNoiseBB, which has received funding from the European Union's Horizon 2020 research and innovation programme under grant agreement No. 690714.

This work was granted access to the HPC resources of CINES under an allocation made by GENCI (French National Computing Center).

The authors are grateful to Sébastien Deck and Nicolas Renard from the Advanced Modeling Turbululence Simulation team in ONERA for the fruitful discussion and their help on the ZDES.

\section{REFERENCES}

[1] Moreau, A., and Enghardt, L., 2009. "Ranking of Fan Broadband Noise Sources based on an Experimental Parametric Study". In 15th AIAA/CEAS Aeroacoustics Conference (30th AIAA Aeroacoustics Conference), p. 3222.

[2] Polacsek, C., Daroukh, M., François, B., and Barrier, R., 2020. "Turbofan Broadband Noise Predictions Based on a ZDES Calculation of a Fan-OGV Stage". In Forum Acusticum, Lyon (France).

[3] Deck, S., 2012. "Recent Improvements in the Zonal Detached Eddy Simulation (ZDES) Formulation”. Theoretical and Computational Fluid Dynamics, 26(6), pp. 523-550.

[4] Bonneau, V., Polacsek, C., Castillon, L., Marty, J., Gervais, Y., and Moreau, S., 2016. "Turbofan Broadband Noise Predictions using a 3-D ZDES Rotor Blade Approach". In 22nd AIAA/CEAS Aeroacoustics Conference, p. 2950.

[5] Leonard, T., Sanjose, M., Moreau, S., and Duchaine, F., 2016. "Large Eddy Simulation of a Scale-Model Turbofan for Fan Noise Source Diagnostic". In 22nd AIAA/CEAS Aeroacoustics Conference, p. 3000.

[6] Arroyo, C. P., Leonard, T., Sanjosé, M., Moreau, S., and Duchaine, F., 2019. "Large Eddy Simulation of a Scale-
Model Turbofan for Fan Noise Source Diagnostic". Journal of Sound and Vibration, 445, pp. 64-76.

[7] Hah, C., and Romeo, M., 2017. "LES Investigation of Wake Development in a Transonic Fan Stage for Aeroacoustic Analysis". In 23rd International Symposium on Air Breathing Engines (ISABE).

[8] Meyer, R., Hakansson, S., Hage, W., and Enghardt, L., 2019. "Instantaneous Flow Field Measurements in the Interstage Section between a Fan and the Outlet Guiding Vanes at Different Axial Positions". In Proceedings of 13th European Conference on Turbomachinery Fluid Dynamics and Thermodynamics, Lausanne, Switzerland, April, pp. 8-12.

[9] Cambier, L., Heib, S., and Plot, S., 2013. "The Onera elsA CFD Software: Input from Research and Feedback from Industry". Mechanics \& Industry, 14(3), pp. 159-174.

[10] Deck, S., Gand, F., Brunet, V., and Ben Khelil, S., 2014. "High-Fidelity Simulations of Unsteady Civil Aircraft Aerodynamics: Stakes and Perspectives. Application of Zonal Detached Eddy Simulation". Philosophical Transactions of the Royal Society A: Mathematical, Physical and Engineering Sciences, 372(2022), p. 20130325.

[11] Riéra, W., Marty, J., Castillon, L., and Deck, S., 2016. "Zonal Detached-Eddy Simulation applied to the TipClearance Flow in an Axial Compressor". AIAA Journal, pp. 2377-2391.

[12] Spalart, P., and Allmaras, S., 1992. "A One-Equation Turbulence Model for Aerodynamic Flows". In 30th aerospace sciences meeting and exhibit, p. 439.

[13] Spalart, P. R., Deck, S., Shur, M. L., Squires, K. D., Strelets, M. K., and Travin, A., 2006. "A New Version of Detached-Eddy Simulation, Resistant to Ambiguous Grid Densities". Theoretical and Computational Fluid Dynamics, 20(3), May, p. 181.

[14] Posson, H., and Roger, M., 2007. "Parametric Study of Gust Scattering and Sound Transmission through a Blade Row". In 13th AIAA/CEAS Aeroacoustics Conference (28th AIAA Aeroacoustics Conference), p. 3690.

[15] Hussain, A. K. M. F., and Reynolds, W. C., 1970. "The Mechanics of an Organized Wave in Turbulent Shear Flow". Journal of Fluid Mechanics, 41(2), pp. 241-258.

[16] Gand, F., 2012. "Zonal Detached Eddy Simulation of a Civil Aircraft with a Deflected Spoiler". AIAA journal, 51(3), pp. 697-706.

[17] Verrière, J., Gand, F., and Deck, S., 2016. "Zonal DetachedEddy Simulations of a Dual-Stream Jet”. AIAA Journal, pp. 3176-3190.

[18] Mockett, C., Fuchs, M., Garbaruk, A., Shur, M., Spalart, P., Strelets, M., Thiele, F., and Travin, A., 2015. "Two NonZonal Approaches to accelerate RANS to LES Transition of Free Shear Layers in DES". In Progress in hybrid ransles modelling. Springer, pp. 187-201. 